\title{
Understanding of the Sung-Rye-Moon Roof Structure and implementation of the traditional Bracket-set Design Modules for BIM tools
}

\author{
Soo-Hoon Park ${ }^{\dagger}$, Eunyoung Ahn ${ }^{++}$
}

\begin{abstract}
Roof structure of the traditional buildings in the Northeast Asia region including Korea contains the most complicated and crucial components of the building and therefore the issues such as cost down, productivity and the attempt to combine the traditional building methodology with contemporary building technology turn out to be vital to the survival of the old yet disconnected traditional building industry. One of the distinctive modern building technologies is handling life ${ }^{-}$cycle building information by constructing virtual buildings using BIM, building information modeling tools. In this paper we follow a procedure to implement some of the design modules to be applied in BIM tools which are platforms for constructing virtual building models. We focus on Gong-po components namely the bracket-sets which are the essential part that connects the middle parts to the top parts (the roof structure) which are considered to be the most elaborate parts of the traditional buildings. The target building to work with in this paper is the Sung-Rye-Moon which has special cultural and social meanings nowadays and we tested our understanding and the design modules such as the bracket-sets by constructing a virtual building model of Sung-Rye-Moon.
\end{abstract}

Key words: bracket-set (Gong-po), design module, BIM, Sung-Rye-Moon.

\section{INTRODUCTION}

Traditional wood structures in North-East Asian region have been the central issues in traditional building industry, however the modern architectural design tools, mostly BIM namely Building Information Modeling tools such as ArchiCAD and Revit, have not provided any sufficient means to work with these components. Those tools are mainly focused on modern building components $1 u^{-}$ crative in building industry such that it becomes

※ Corresponding Author: E.Y. Ahn, San 16-1, DeokmyungDong, Deajeon-City. Korea: TEL :+82-42-821-1750, FAX : +82-42-821-1595, E-mail : aey@hanbat.ac.kr Receipt date: Oct. 31, 2011, Revision date: Dec. 19, 2011 Approval date: Dec. 21, 2011

${ }^{+}$Major in Architecture, Hanbat National University, Korea

(E-mail: soohoon@hanbat.ac.kr)

${ }^{++}$Div. of Information Communication \& Computer Engineering, Hanbat National University, Korea exceptionally hard to use contemporary building designing tools in designing and modeling traditional buildings and components.

This paper concerns about traditional wooden roof structure and we focus on the GongPo components namely the bracket-sets. We are examining especially the roof structure because this top-part of the traditional wood building contains the most complicated wooden compositions comparing with the middle- and the bottom parts. We focus on GongPo components such that these elements are regarded as the single most important and the most diverse elements in the roof structure of the traditional Northeast Asian buildings. Understanding the GongPo components and implementing them in the current BIM platform such as ArchiCAD as a virtual building with integrated design database would achieve those educational as well as cultural and industrial aims surrounding virtual building 
construction and contents making of traditional architecture.

The target building we chose for this paper is the Sung-Rye-Moon(SRM) a.k.a. the Grand South Gate. The Grand South Gate, along with those Grand Gates in Gae Sung and Pyong Yang cities, is known as one of the oldest Gate building in Korea such that it has significant historical values since its construction in 1398 and many repairs including those in 1449 and 1479. This SRM or GSG is a rare case in the world in the sense that the building itself is the first national treasure and it also reminded us the importance of the protection of cultural heritages remembering the tragic $\mathrm{de}^{-}$ struction by fire in $10^{\text {th }}$ of Feb. 2008. We constructed the virtual building of Sung-Rye-Moon based on the detailed survey data by SamSung Architects[1].

\section{TRADITIONAL WOOD STRUCTURE}

The composition and the components of the traditional wood structure of the Northeast Asian buildings are quite characteristic in many ways compared to the modern or wooden structures in many regions.

\subsection{The Roof Structure}

Since the $10^{\text {th }}$ century, Northeast Asian buildings are considered to be understood as a trilogy of three parts (the script on wood structure, 10C): the bottom part (the platform), the middle part and the top part (the roof structure above the connecting beams). The middle and the top part are divided along with the connecting beams. The bottom part is the least diverse and mostly the stone structures. The most diverse, the biggest, the broadest, the heaviest and structurally the most complicated areas are the top part, i.e. the roof structure. Understanding the roof structure of the traditional building, therefore, are essential in terms of knowledge on the traditional wood construction.

\subsection{Principles and Structure Prototypes}

G.M. Lee[2] suggests two main principles in understanding wood structuring in terms of supporting the upper elements.

- Stacking principle:

- Inserting principle:

Three main elements in wood structuring are the horizontal, the vertical and diagonal elements. Stacking principle is normally seen from structures where the horizontal elements are stacked on top of the vertical elements. Inserting principle is normally seen in joints where two elements are penetrated/inserted each other as shown in Fig. 1(a).

The stacking principles are basically noticed from the purlins and the crossbeams on the $\mathrm{col}^{-}$ umns while the inserting principles are witnessed in the cases such as architraves. Stacking principles are considered to be applied to the main wood structures because of its superior load transfer capability. On the other hand the inserting principle show superiority on its resistance toward lateral loads and forces in the sense that members in a component are not easily separated and maintaining the form therefore this inserting principle is applied where the position fixing/ locking up characteristics are more important than vertical load transfer.

These two principles are more clearly contrasted in terms of two main structural prototypes such as the purlin structure prototype and the rafter

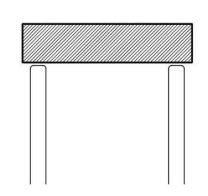

Fig. 1. (a) The principle[2].
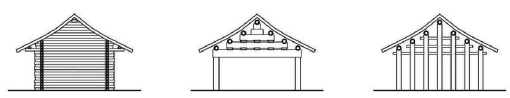

Fig. 1. (b) The purlin-structure \& the prototypes (left to right) [2]

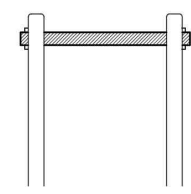

inserting

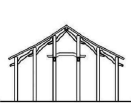

raster-structure 
structure prototype matching to stacking and inserting principles respectively. The Rafter structure prototype is formed with diagonal members of the components and the purlin structure prototype is the combination of the horizontal and vertical members of the components as shown in Fig. 1(b).

\subsection{The Bracket-set (GongPo)}

The bracket-set (GongPo) is considered as one of the most characteristic components in the Northeast Asian architecture and we witness there have been too much focuses given to these components which simply connect the lintel, the column and the roof structure. The more frequent use and the more complicated the bracket-sets are applied, the more sublime the building becomes and the change of GongPo components far exceeds the speed of changing the building type such that the use and the development of GongPo became the key component in examining and distinguishing traditional buildings.

The main function of the GongPo is to protrude the eaves to the front. Protruding eaves of the roof structure has been quite a critical issue in most of the Northeast Asian countries and the patterns are analyzed in terms of three categories as shown in Fig. 2. The first is (1) to protrude the rafters (and other supporting diagonal members) to the limit. The second is (2) to support the protruded eaves using the horizontal beams through columns. The last method is (3) to support eaves using the bracket-sets (GongPo). GongPo components are later integrated with well-shaped horizontal and vertical crisscrossing structure to be evolved into more complicated patterns.

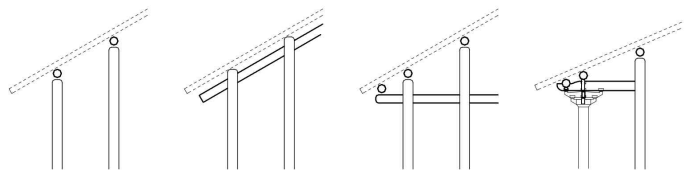

Fig. 2. Methods of protruding eaves [2].

\section{THE ROOF STRUCTURE OF THE SUNG-RYE-MOON}

English naming summary of elements in the roof structure of the Sung-Rye-Moon is shown in Fig. 3. The loads of the top part of the building are transferred to the middle part where the bracket-sets are used to cope with many functionalities as follows:

- Cantilever eaves:

- Connecting the rafter structures/ inserting structure-sets to the purlin structures/ stacking structure-sets:

- Forming the Façade of the building:

The three directions in the traditional buildings are the column direction (vertical), the crossbeam direction (horizontal) and the purlin direction (horizontal and orthogonal to the beam direction). A typical section of the building is conceived in terms of the column and the beam direction. The extension of the building is mostly to the purlin direction

The purlin direction is important not only because it is the building-extension direction but also in the sense that it is the approach and the access

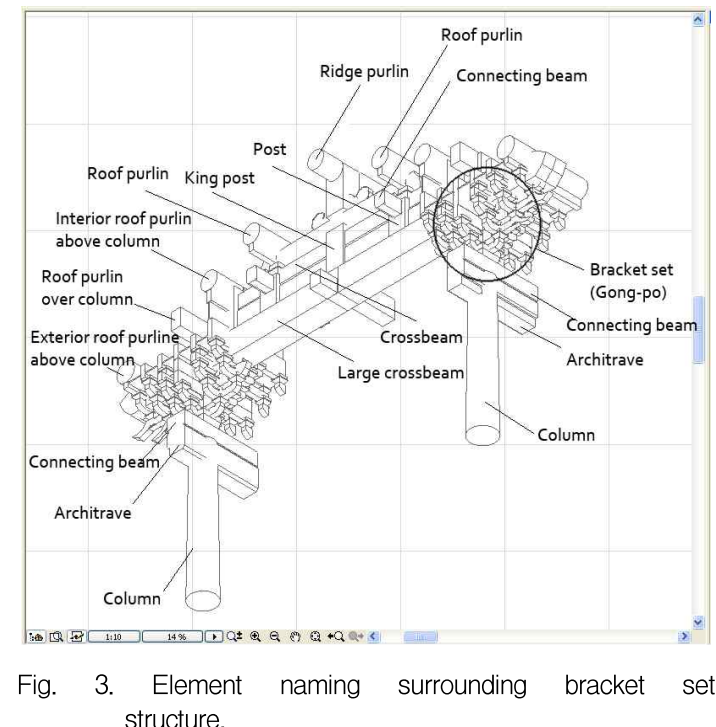


to the building. That is why the bracket-sets are elaborated in such a way that the numbers and the composition style of the bracket-sets are increased to form more luxurious façade of the building. The bracket-sets also functions as the position fixer that locks up the rafter structure sets containing many diagonal members to the stacking structure sets composed of much static and safer horizontal and vertical members[4]. The bracket sets are also functioning as the structural support for the cantilever eaves.

\subsection{The bracket-sets of the SRM}

As shown in Fig. 4, SRM has the bracket-sets with the style of early Chosun Dapo family where the bracket sets are positioned not only on top of columns but also in the intervals of the columns.

The bracket sets of the SRM as in Figure 5 shows $5^{-}$-steps (5 po) where the JeGong and the ChumCha (bracket) are crisscrossing to form a well-shaped structure component. The naming and the numbering of this crisscrossing members of the bracket set components are roughly shown in Figure 5. The horizontal members in the crossbeam direction are named as the JeGong which stack one after another. The other horizontal members in the purlin/lintel direction are named as the ChumCha (the brackets) which are positioned along with axis on the center and/or stepped out off the center of the column. These two horizontally crisscrossing stacking members compose the bracket sets and they are used to determine the scale and typology of the bracket sets (GongPo).

In Sung-Rye-Moon, there are several variations of the bracket sets showing small differences in
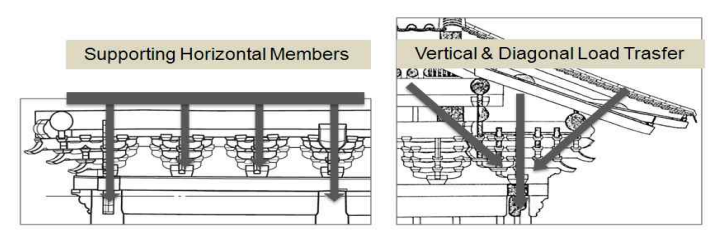

Fig. 4. Structure load transfer at the bracket sets.

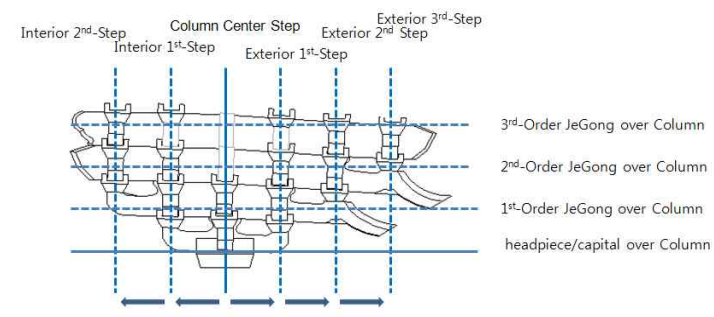

Fig. 5. The numbering of ChulMok (Steps).

their positioning in both floors as shown in Fig. 6. The main difference between the bracket- sets in the lower floor and the upper floor is the number of steps where each JeGong supports the brackets (ChumCha) on a number of vertical axis on and/or off the center of columns. Starting from the axis on the column, the lower components have exterior two-steps and interior two-steps of the brackets (ChumCha) on the multi-story JeGong while the upper level components have more steps toward outside containing exterior three steps and interior two steps of the brackets (ChumCha). The exterior end of each JeGong is decorated with cow-tongue shaped SueSeo.

The well-shaped composition of the bracket sets forms the inverted pyramid assembly where the JeGong in the beam direction horizontal members and the brackets (ChumCha) in the purlin/lintel direction horizontal members are increasing in its sizes and number of joints as it stacks on top of each other. The disassembled members of the bracket set in SRM are shown in Fig. 7 which dis-
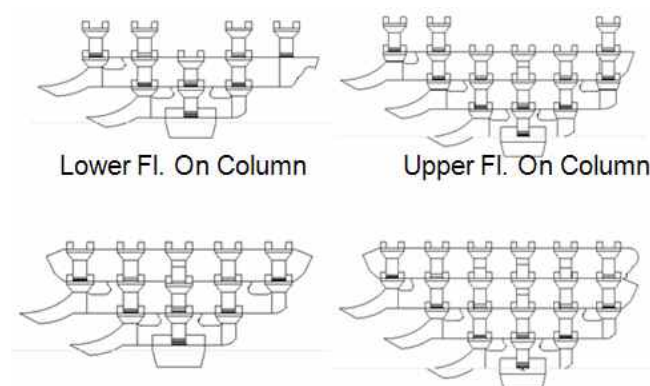

Lower FI. Between Column

Upper FI. Between Column

Fig. 6. The variation of the bracket sets in the SRM [1] 
tinguishes the big and small brackets (ChumCha) according to their positioning.

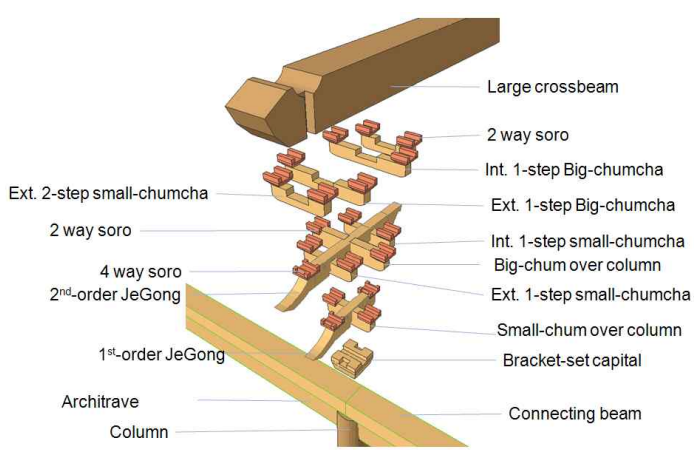

Fig. 7. Bracket-set components and composition.

\subsection{The layout of the bracket-sets in the SRM}

The layout of the bracket sets in the SRM building shows similar yet different patterns in lower and upper floor levels. The measuring concept for a floor plan space is called Kan which counts the numbers between the columns. The spatial layout of the SRM building shows front 5 -Kan and side 2-Kan for both upper and lower levels comprising the floor areas of $173.46 \mathrm{~m}^{2}$ (52.47Pyong) and 137.86 $\mathrm{m}^{2}$ (41.70 Pyong) respectively. The bracket sets for both upper and lower levels consists of three types namely On-column po, Between-column po and Corner-column po where po means the bracket set. There are 38 (upper) and 46 (lower) sets of GongPo in each level as shown in Fig. 8 and 9.

\subsection{The composition of the Bracket-set}

The bracket set is the building device that supports one or multiple set of purlins (Dori) delivering the load of the roof structure toward the columns It is basically a structural element that holds the upper rafter structure sets and helps the diagonal rafter members to be protruded to their limit to form cantilever eaves.

Fig. 10 shows views on a bracket-set. The stacking of the bracket-set members starts with the headpiece (the capital) which is positioned on

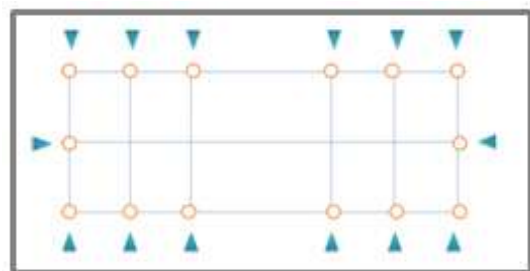

(a) On Column GongPo layouts
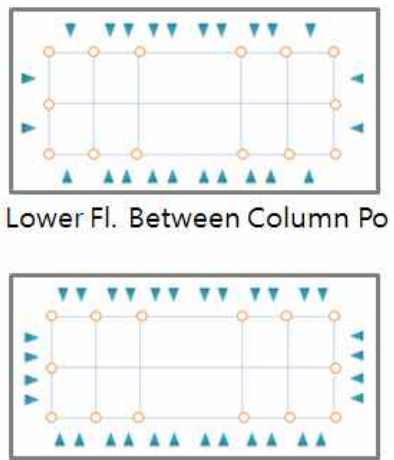

Upper Fl. Between Column Po

(b) Between Column

Fig. 8. The Gongpo Layouts [1].

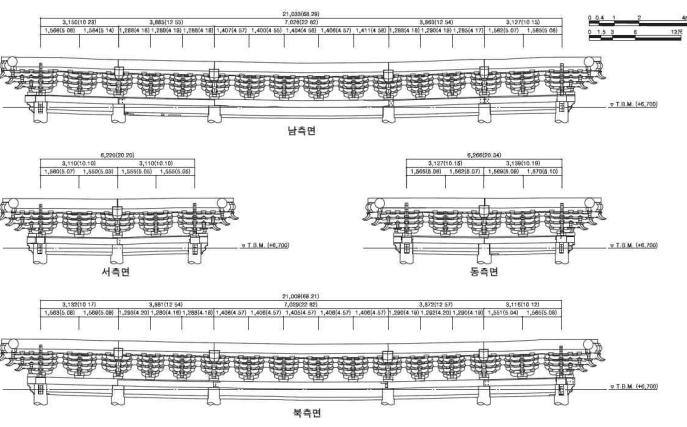

Fig. 9. The layout of Gongpo in the upper level [1]

top of the connecting beam housing the $1^{\text {st }}$ JeGong where a set of brackets are placed crisscrossing to each other. The lower brackets are smaller and the bracket holds, on the tip of its arms, the SoRo which functions and shapes basically similar to the headpiece (the capital) housing the upper brackets in either 2-way or 4-way directions. On the top part of the highest JeGong places the large crossbeam on which one or more purlins are supported as shown in Fig. 10. The invert-Pyramid shapes of the bracket-set components are the joining 


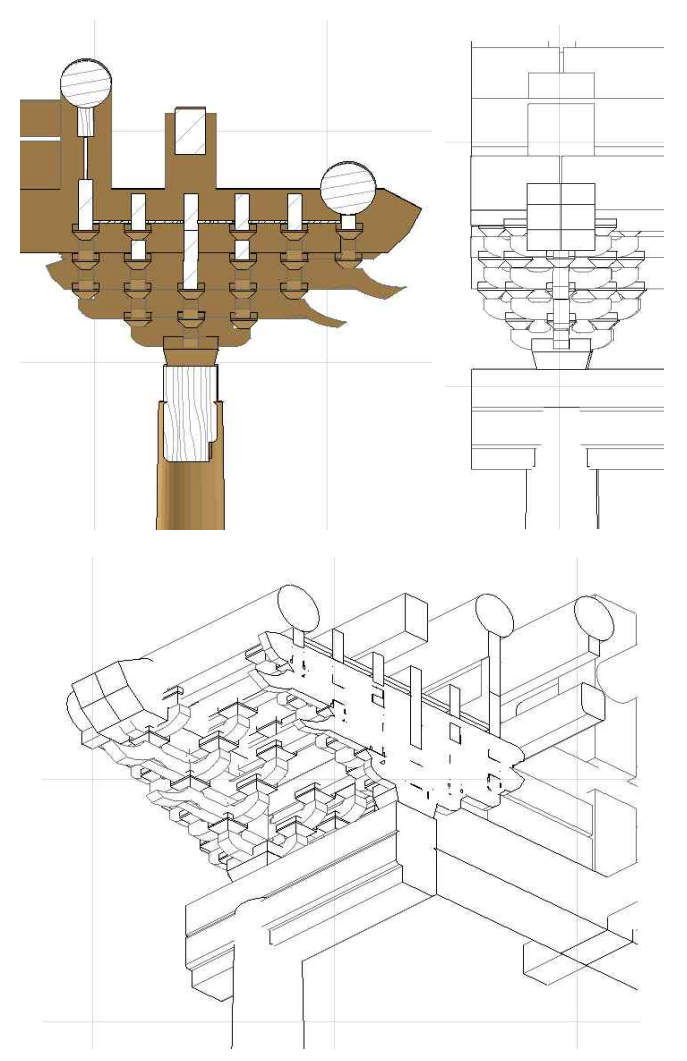

Fig. 10. Various views on a bracket-set in SRM.

points where roof structure meets floor spaces. The space measuring concept for the roof structure is named as Gha which means in between the purlins as a diagonal bridge and those of the floor spaces are Kan which means in between the columns as shown in Fig. 11. The bracket sets function as the joining point of these two genetically heterogeneous structure sets.

\section{DESIGN MODULES AND BIM MO- DELING}

\subsection{Gong-po design modules}

This paper illustrates the development of design modules for the bracket sets consists of typical members such as JeGong, brackets, headpiece and SoRo. The diagram for the implementation process is shown in Fig. 12.

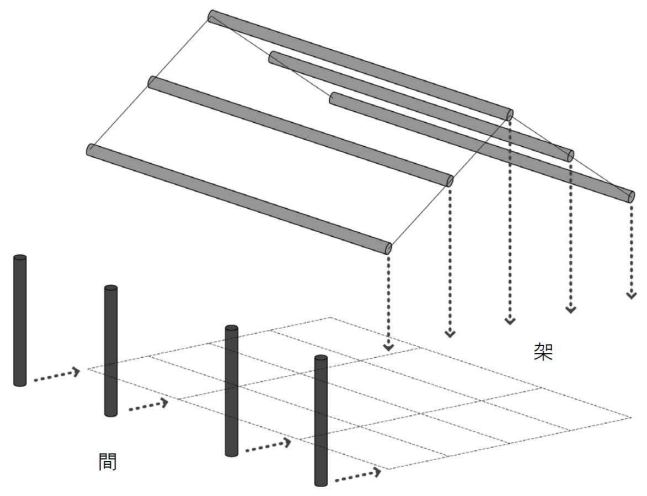

Fig. 11. The combination of Kan \& Gha [2].

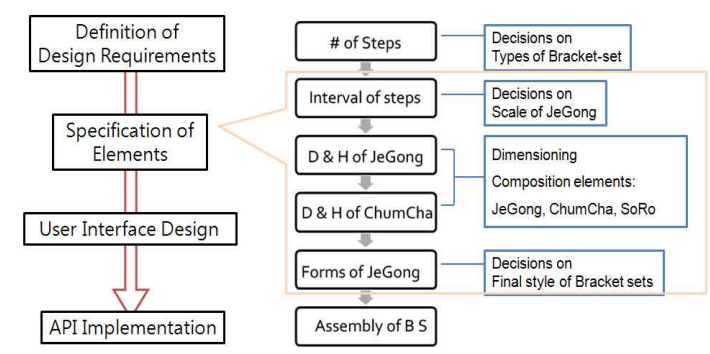

Fig. 12. Implementation of the bracket-set design module.

(1) Decisions on \# of Steps (ChulMok). The bracket set types are decided based on these numbers.

(2) Decisions on the interval of steps \& the brackets, size of JeGong

(3) Decisions on the depth and the height of JeGong

(4) Decisions on the depth and the height of the brackets

(5) Decisions on end forms of JeGong members

(6) Assembly of the bracket sets using these design decisions

Followings are the brief procedure description for implementing the traditional building design modules for BIM tools as illustrated in Fig. 13.

(1) Definitions on design requirements of members based on drawings and/or detailed surveys.

(2) Specification of elements and scripts on element forms.

(3) Parametric design module implementation using GDL. 
(4) User interface implementation using API.

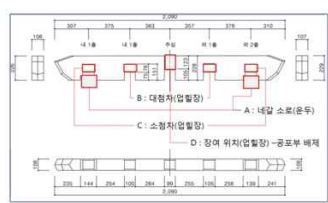

Design requirements

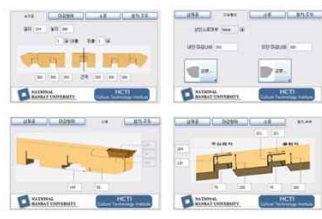

User interface using GDL \& API

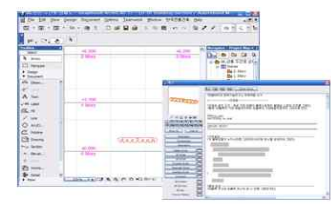

Scripts on elements

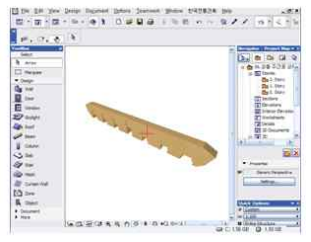

Members in the BIM tool
Fig.13. Design module implementation for a large crossbeam.

\subsection{Implementation of the bracket sets}

Typical members of the bracket sets such as headpiece, 4-way and 2-way SoRo, brackets and JeGong are implemented using the design modules in the BIM tool called Graphisoft ArchiCAD version 12 on Windows as in Fig. 14.

The 3D forms of the SRM building are handled in the BIM tool platform called ArchiCAD version 12 where various traditional building design modules are implemented[5][6]. All these numeric data for each design module is based on the detailed survey and measure report of SRM in 2006. Patterns such as DanChong coloring on the elements of roof eaves and columns are separately produced based upon photographs of many sources and they are redrawn using the tools such as photoshop and mapped onto each design module as shown in Fig. 15 and 16.
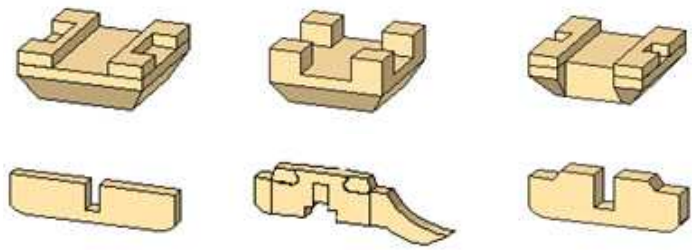

Fig. 14. Typical members of the bracket sets.

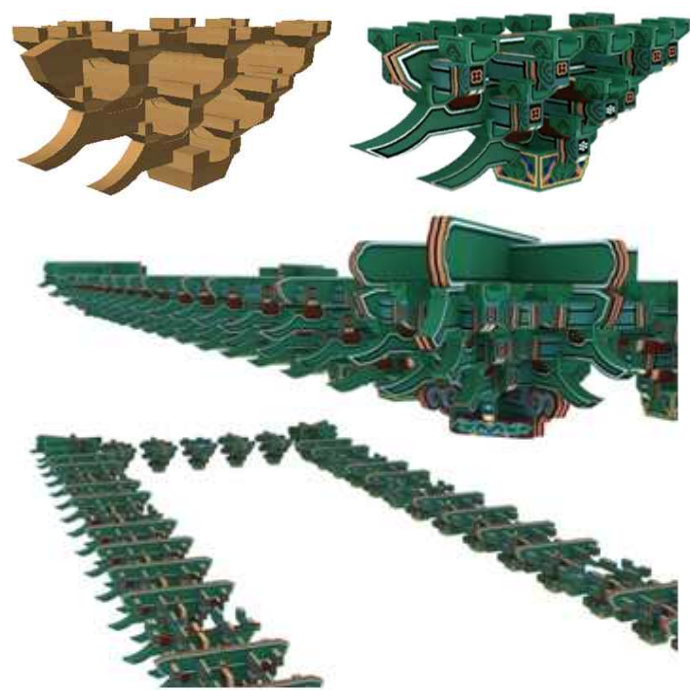

Fig. 15. Modeling and image mapping on the Bracket sets.

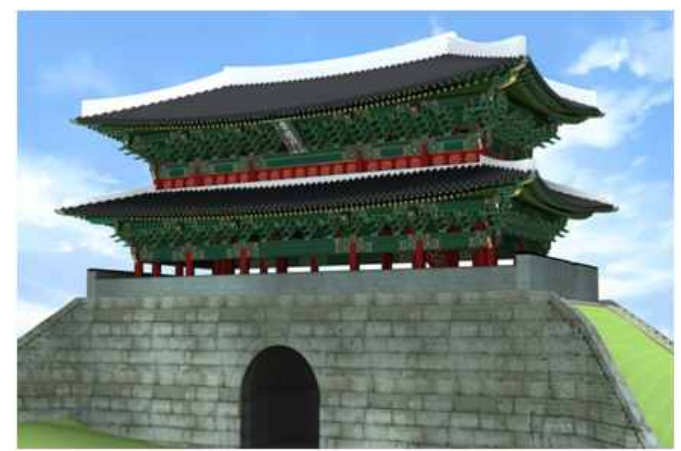

Fig. 16. Modeling and rendering image of the SRM.

\section{CONCLUSION}

Traditional buildings in the Northeast Asian region are huge in numbers and yet the cutting-edge building modeling tools are not taking this matter seriously in embracing them as their subjects. We therefore tried to fill this gap of current building modeling tools and these alienated subjects by implementing design modules for building components suited for the BIM tools such as ArchiCAD version 12. Modeling a component is one thing and understanding the traditional building and constructing virtual building models in their context are another matter so that we needed proper 
knowledge, concepts, measures, names and assembly knowhow in order to avoid distinctive mistakes.

The models along with all the construction information including sizes, unit costs, materials and positions could turn out to be valuable sources of the cultural contents such as this SRM example. We could measure individual members in developing modules. We could assemble building devices as meaningful components. We could assemble the roof structures, the floors and the stone structures respectively and view them separately. We could also disassemble them, slice them to make section views, count individual members and calculate their material volumes. All these design and construction information has the possibility to be $\mathrm{ex}^{-}$ amined further and with more intention of future applications.

\section{ACKNOWLEDGEMENT}

This research was supported by Contents Industry Technology Support Program through Ministry of Culture, Sports and Tourism(MCST) and Korea Creative Content Agency(KOCCA) (2011).

\section{REFERENCES}

[1] SamSung Architects, Sung Rye Moon detailed survey and analysis report, DongA, 2006.

[2] G.M. Lee, The Building Principles and Typology of Roof Structure in the East Asian Wood Architecture, Ph.D. Thesis, Seoul National University, 2009.

[3] S.-L.Ryoo, "A Study on an Alternative Idea of 'Opinion of Roof Style \& Structure Changes of Sung-Rye-Mun' following the first construction in Cho-Sun Dynasty," Architectural Institute of Korea, Vol.25, No.12, pp. 209-220, 2009.
[ 4 ] J.-K. Hwang, J.-W. Kwak, and J.-H. Kwak, "Resisting Capacity of Korean Traditional Wooden Structural Systems Subjected to Static Loadings," Structural Engineering and Mechanics, Vol.30, No.3, pp. 297-316, 2008.

[5] D. Nicholson-Cole, The GDL Cookbook 4 The source of all that is good in GDL and ArchiCAD Tips and Tricks, Marmalade Graphics, 2004.

[6] Graphisoft, ArchiCAD 12 GDL Reference Manual, Graphisoft Vol 4, pp. 31-114, 2009

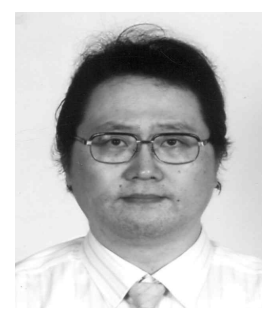

\section{Soo-Hoon Park}

He is an associate Professor in Architecture major at Hanbat National University in Daejeon, Korea. He received BE, ME de ${ }^{-}$ grees in Architecture from Seoul National University, Korea in 1987 and in 1989, M. Arch degree in UCLA, USA in 1994 and Ph. D. in Architecture at University of Sydney, Australia in 1999. Dr. Park's area of interest includes Design Computing and Cognition, BIM and Computer Graphics.

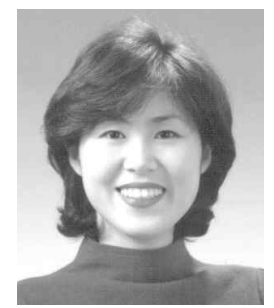

\section{Eunyoung Ahn}

She is an associate Professor in Department of Information Comm \& Computer Eng. at Hanbat National University in Daejoen, Korea. She received BS and MS degrees in computer science from the Dongguk University, Korea, in 1989 and in 1991, respectively and $\mathrm{Ph}$. D in Computer Science from the Dongguk University, Korea, in 2000. She is the author of Computer Animation (Yung-jin press). Dr. Ahn's area of interest includes Computer Graphics, Virtual Reality, U-learning, 3D reconstruction, and Culture Technology. 2009. 